\title{
FRET-Based Optical Assay for Monitoring Riboswitch Activation
}

\author{
Svetlana Harbaugh, ${ }^{\dagger}$ Nancy Kelley-Loughnane, ${ }^{\dagger}$ Molly Davidson, ${ }^{\dagger}$ Latha Narayanan, ${ }^{\dagger}$ \\ Sandra Trott, Yaroslav G. Chushak, ${ }^{\ddagger}$ and Morley O. Stone ${ }^{*, \dagger}$ \\ Applied Biotechnology Branch, Human Effectiveness Directorate, Air Force Research Laboratory, \\ Wright-Patterson Air Force Base, Dayton, Ohio 45433, and Biotechnology HPC Software Applications Institute, \\ Telemedicine and Advanced Technology Research Center, U.S. Army Medical Research and \\ Material Command, Fort Detrick, Maryland 21702
}

Received October 3, 2008; Revised Manuscript Received December 23, 2008

\begin{abstract}
Riboswitches are regulatory RNAs located in the 5'-untranslated region of mRNA sequences that recognize and bind to small molecules and regulate the expression of downstream genes. Creation of synthetic riboswitches to novel ligands depends on the ability to monitor riboswitch activation in the presence of analyte. In our work, we have coupled a synthetic riboswitch to an optical reporter assay based on fluorescence resonance energy transfer (FRET) between two genetically encoded fluorescent proteins. The theophylline-sensitive riboswitch was placed upstream of the Tobacco Etch Virus (TEV) protease coding sequence. Our FRET construct was composed of eGFP and a nonfluorescent yellow fluorescent protein mutant called REACh (for resonance energy-accepting chromoprotein) connected with a peptide linker containing a TEV protease cleavage site. Addition of theophylline to the $E$. coli cells activates the riboswitch and initiates the translation of mRNA. Synthesized protease cleaves the linker in the FRET-based fusion protein causing a change in the fluorescence signal. By this method, we observed an 11-fold increase in cellular extract fluorescence in the presence of theophylline. The advantage of using an eGFP-REACh pair is the elimination of acceptor fluorescence. This leads to an improved detection of FRET via better signal-to-noise ratio, allowing us to monitor riboswitch activation in a wide range of analyte concentrations from 0.01 to $2.5 \mathrm{mM}$.
\end{abstract}

\section{Introduction}

Living organisms have developed the ability to control their gene expression patterns in response to changes in the extracellular and intracellular environment. While traditionally gene regulation has been ascribed to proteins, recently, a new class of RNA molecules that regulate gene expression, riboswitches, have been discovered. ${ }^{1-4}$ Riboswitches are RNA sequences located in the $5^{\prime}$ untranslated region $\left(5^{\prime}\right.$-UTR) of mRNA that can serve as molecular switches able to regulate the level of gene expression through the conformational changes caused by binding to a specific metabolite. Riboswitches contain two structural domains: an aptamer domain that senses and binds to a metabolite and an expression platform that controls the expression of a downstream gene by changing its conformation in response to the ligand-induced changes in the aptamer domain. $^{5}$

In addition to natural riboswitches found in living organisms, a variety of synthetic riboswitches that respond to different small molecules have been developed. ${ }^{6-9}$ The most common method for the creation of a synthetic riboswitch is cloning of an aptamer sequence into the $5^{\prime}$-UTR of a given mRNA. Aptamers have been generated for a variety of ligands including small organic molecules, peptides, and aminoglycosides among others. ${ }^{10}$ The advantage of such engineered riboswitches is that they offer a way to control gene expression via non-natural molecules, for example, drug compounds.

* To whom correspondence should be addressed. E-mail: morley.stone@ wpafb.af.mil.

Air Force Research Laboratory, Dayton, OH.

*USAMRMC, Fort Detrick, MD.
The development of new synthetic riboswitches depends on our ability to monitor the activity of riboswitches in living cells. Approaches based on fluorescence spectroscopy have demonstrated high efficiency and sensitivity in the visualization of gene expression patterns. ${ }^{11,12}$ In one example, an engineered riboswitch was cloned directly upstream of the green fluorescent protein (GFP) gene and activation of the riboswitch in the presence of ligand was analyzed by monitoring the intensity of fluorescence, ${ }^{13,14}$ which is proportional to the number of GFP molecules. In an effort to amplify the signal response beyond GFP, we have coupled the theophylline riboswitch to an enzymatic cascade that cleaves an optically engineered protein substrate. The protein substrate is a fluorescence resonance energy transfer (FRET) construct between two fluorescent protein conjugates. ${ }^{15}$ The first protein in the pair acts as the electron energy donor and the second represents an electron energy acceptor. FRET involves the distance-dependent energy transfer from an excited donor molecule to a lower energy acceptor and is measured in terms of spectral intensity changes of the emission signal. The efficiency of this process varies with the sixth power of the intermolecular separation and is usually described by means of the Förster radius ${ }^{16}$ (a distance at which coupling efficiency reaches $50 \%$, typically within the range of 2-7 nm). Our FRET construct is based on the enhanced green fluorescent protein (eGFP) and a nonfluorescent mutant of the yellow fluorescent protein (YFP) called resonance energyaccepting chromoprotein (REACh). ${ }^{17}$ Because REACh is a "dark" protein, it acts as a quencher for the donor fluorescence by the FRET mechanism. The advantage of using REACh in the FRET construct is the elimination of acceptor fluorescence, providing better quantitative analysis of the output signal based 

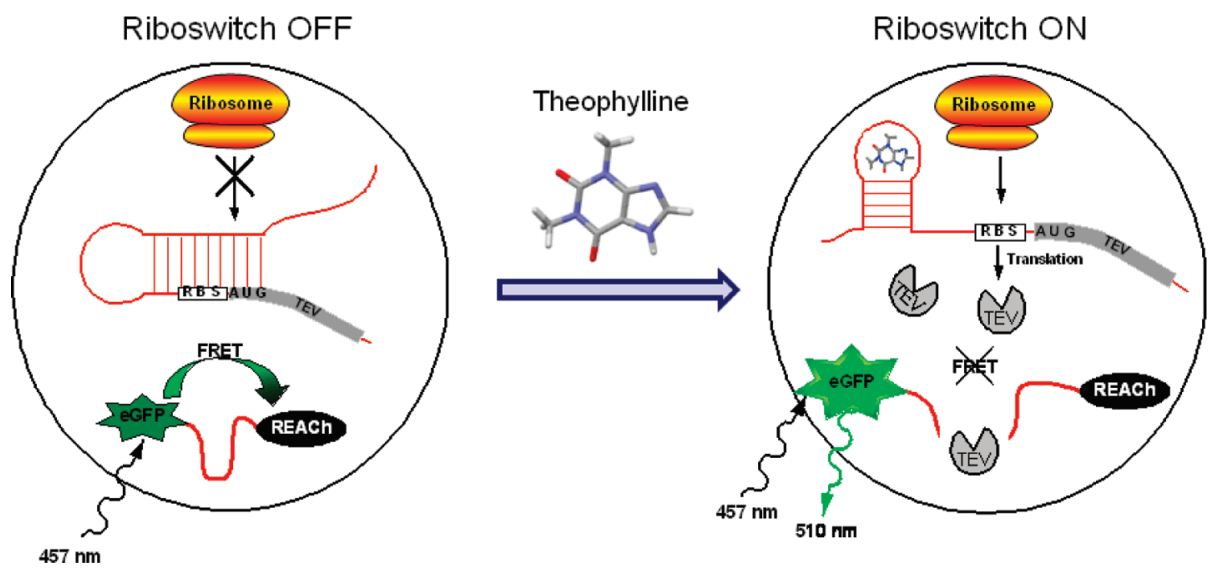

Figure 1. FRET-based reporter assay for in vivo monitoring of theophylline-responsive riboswitch activation. In the absence of theophylline, extensive pairing of riboswitch with ribosome binding site (RBS) prevents translation of TEV protease; riboswitch is in "off" state. Upon theophylline binding, the RBS becomes unpaired, thus allowing for the translation of the TEV protease mRNA; riboswitch is in "on" state. The translated TEV protease cleaves the FRET-based protein construct, yielding an increase in fluorescence emission at $510 \mathrm{~nm}$ upon excitation at $457 \mathrm{~nm}$.

on riboswitch activity. The superior donor eGFP is able to be linked to the modified YFP due to the elimination of the spectral overlap that would normally encumber analysis. The low optical starting point (low background) allowed for improved sensitivity in our cellular system due to the spectrally "clean" release of eGFP chromophore.

For enzymatic amplification of signal, we chose Tobacco Etch Virus (TEV) protease. TEV protease was chosen due to the stringent sequence specificity required for proteolytic activity. ${ }^{18}$ TEV protease has been well characterized with a seven amino acid recognition site composed of ENLYFQG/S, and cleavage occurs with high specificity between QG or QS. Several mutant TEV proteases have been generated that have eliminated its susceptibility to autodigestion which results in lower activity. ${ }^{19}$ Also, directed-evolution studies on mutant TEV proteases identified a variant enzyme with higher yields, increased activity, and increased solubility for expression in $E$. coli. ${ }^{20}$ In an effort to achieve an enzymatic signal cascade (amplification via FRET cleavage), our FRET construct linked eGFP and REACh with a 17 amino acid peptide sequence that included the recognition site for TEV protease. To demonstrate the feasibility of this reporter construct, the synthetic theophylline riboswitch ${ }^{21}$ was placed upstream of the TEV protease gene. This riboswitch was designed to activate expression of TEV protease in E. coli upon binding of the small-molecule theophylline. In the absence of theophylline, an extensive base pairing of the riboswitch with the ribosome binding site (RBS) placed the riboswitch in the "off" state and prevented the translation of TEV protease, as shown in Figure 1. Addition of theophylline changed the conformation of the riboswitch, unpairing the RBS, and initiating the translation of mRNA, thus, the riboswitch was in the "on" state. The resulting synthesized TEV protease cleaves the FRETprotein, causing a change in the fluorescence signal, allowing us to monitor riboswitch activation.

In this work, we have developed a novel optical assay for the readout of riboswitch activation in a low optical background facilitating biological readout and increasing assay sensitivity. The substrate was designed to examine the response of the riboswitch to the addition of ligand and in contrast to conventional FRET assays, using less-sensitive spectroscopic ratio methods, produced a direct optical signal from a quenched background. ${ }^{17}$ With this approach, we have achieved greater temporal control and specificity by developing an enzymatic optical reporting system of riboswitch activation.

\section{Experimental Section}

General Considerations. All plasmid manipulations were performed using the standard cloning techniques, ${ }^{22}$ and the sequences of all constructs have been verified by DNA sequencing at the Plant-Microbe Genomics Facility of The Ohio State University. Purifications of plasmid DNA, PCR products, and restriction enzymes digestions were performed using kits from Qiagen (Valencia, CA). Restriction enzymes (NdeI, BamHI, KpnI, and HindIII) and T4 DNA-ligase were obtained from Invitrogen (Carlsbad, CA). Pfu Ultra DNA-polymerase was purchased from Stratagene (La Jolla, CA). Theophylline, ampicillin, and chloramphenicol were obtained from Sigma (St. Louis, MO). L-(+)Rhamnose monohydrate and dimethylsulfoxide (DMSO) were purchased from Fluka (Switzerland). Synthetic oligonucleotides were supplied by Integrated DNA Technologies (Coralville, IA), and the construct of the synthetic theophylline riboswitch upstream of the TEV protease gene was made by GenScript (Piscataway, NJ). MAX Efficiency DH5 $\alpha$ and One Shot TOP10 competent E. coli cells were acquired from Invitrogen. Difco Luria-Bertani (LB) was purchased from Becton Dickinson and Company (Sparks, MD). The TEV protease gene construct and plasmid pHWG640 were the generous gifts from Dr. Helena Berglund from Karolinska Institute in Stockholm, Sweden and Dr. Josef Altenbuchner from the Institute of Industrial Genetics at the University of Stuttgart, Germany, respectively. The mutant TEV protease was utilized since it showed higher yields, increased activity, and increased solubility from E. coli expression. ${ }^{20}$

The plasmid vector pSAL8.1 was kindly provided by Dr. Justin Gallivan from Emory University, Atlanta, GA. The plasmid vector pIVEX2.3d-eGFP was a generous gift from Dr. Vincent Noireaux from the University of Minnesota.

Plasmid Constructions. Plasmid manipulations were performed using chemically competent MAX Efficiency DH5 $\alpha$ E. coli cells. Full descriptions of primer sequences and plasmid construction techniques are available in the Supporting Information.

Proteolytic Assay in Bacteria. For protein expression, pHWG640: eGFP-TL-REAChHis and a TEV protease expressing plasmid, either pSAL:TEVHis, pSAL:RSTEVHis, or pSAL:RSTEVC151A, were transformed into chemically competent $E$. coli TOP10 cells. Three separate colonies of E. coli TOP10 cells harboring either the positive control (pHWG640:eGFP-TL-REAChHis and pSAL:TEVHis), riboswitch (pHWG640:eGFP-TL-REAChHis and pSAL:RSTEVHis), or negative control (pHWG640:eGFP-TL-REAChHis and pSAL:RSTEVC151A) were picked from LB/agar plates containing ampicillin $(100 \mu \mathrm{g} / \mathrm{mL})$ and chloramphenicol $(25 \mu \mathrm{g} / \mathrm{mL})$ and grown overnight at $37{ }^{\circ} \mathrm{C}$ in separate flasks containing $50 \mathrm{~mL}$ of LB-media supplemented with ampicillin $(100 \mu \mathrm{g} / \mathrm{mL})$ and chloramphenicol $(25 \mu \mathrm{g} / \mathrm{mL})$. A $1.5 \mathrm{~mL}$ aliquot of the overnight cultures was used to inoculate $150 \mathrm{~mL}$ of $\mathrm{LB}$ 
supplemented with ampicillin $(100 \mu \mathrm{g} / \mathrm{mL})$ and chloramphenicol $(25 \mu \mathrm{g} / \mathrm{mL})$. Cells were grown at $37{ }^{\circ} \mathrm{C}$ to an $\mathrm{OD}_{600}$ of $0.4-0.5$ and induced with $0.4 \%$ rhamnose for pHWG640:eGFP-TL-REAChHis expression. After $60 \mathrm{~min}$ of induction, several cultures harboring positive control, riboswitch, or negative control were treated with either $2.5 \mathrm{mM}$ theophylline in DMSO, or an equivalent volume of DMSO for controls with no analyte. Cells were grown at $37{ }^{\circ} \mathrm{C}$ with shaking at $225 \mathrm{rpm}$ for $6 \mathrm{~h}$. Aliquots of $10 \mathrm{~mL}$ culture were collected in $15 \mathrm{~mL}$ Falcon tubes at 0, 60, 120, 180, 240, 300, 360, 420, and $480 \mathrm{~min}$. Cells were harvested by centrifugation at $4000 \times \mathrm{g}$ in Thermo Electron CL3R centrifuge for $10 \mathrm{~min}$ at $4{ }^{\circ} \mathrm{C}$ and cell pellets stored at $-80{ }^{\circ} \mathrm{C}$ for future processing.

The cell pellets were resuspended in $0.4 \mathrm{~mL}$ of lysis buffer $(100$ $\mathrm{mM}$ Tris- $\mathrm{HCl}, \mathrm{pH} 8.0,150 \mathrm{mM} \mathrm{NaCl}, 1 \mathrm{mM} \mathrm{MgCl}_{2}$ ) and incubated on ice for $30 \mathrm{~min}$. The buffer was supplemented with lysozyme $(0.1 \mathrm{mg} / \mathrm{mL})$, benzonase nuclease $(10 \mu \mathrm{L} / 10 \mathrm{~mL})$, and the protease inhibitor iodoacetamide $(8 \mathrm{mM})$. The samples were clarified by centrifugation at $14000 \times \mathrm{g}$ for $30 \mathrm{~min}$ at $4{ }^{\circ} \mathrm{C}$ in an Eppendorf $5417 \mathrm{R}$ centrifuge (Fisher Scientific, Fairlawn, NJ). The clarified cellular extracts were examined using spectrofluorimetric analyses.

Spectral Analysis. Emission spectra of recombinant eGFP-TLREACh1 were acquired by utilizing Molecular Devices Spectra Max M5 spectrophotometer. Upon excitation at $457 \mathrm{~nm}$, the constructed eGFP-TL-REACh FRET pair shows an insignificant emission peak at $510 \mathrm{~nm}$ for the donor eGFP. Proteolytic cleavage of FRET protein by TEV protease resulted in an increase of eGFP emission at $510 \mathrm{~nm}$. The emission at $510 \mathrm{~nm}$ was used to quantify the activity of the riboswitch.

Kinetic Assay. E. coli TOP10 cells harboring pHWG640 (empty plasmid) and a TEV protease expressing plasmid, either pSAL:TEVHis or pSAL:RSTEV, were grown at $37^{\circ} \mathrm{C}$ in LB medium containing 100 $\mu \mathrm{g} / \mathrm{mL}$ ampicillin and $25 \mu \mathrm{g} / \mathrm{mL}$ chloramphenicol. The expression cultures were inoculated from overnight cultures and cells were grown at $37{ }^{\circ} \mathrm{C}$ to an $\mathrm{OD}_{600}$ of $0.4-0.5$ and $0.4 \%$ rhamnose was added. After $60 \mathrm{~min}$, cultures harboring positive control and riboswitch were treated with either $2.5 \mathrm{mM}$ theophylline in DMSO or an equivalent volume of DMSO for controls with no analyte. Cells were grown at $37{ }^{\circ} \mathrm{C}$ with shaking at $225 \mathrm{rpm}$ for $3 \mathrm{~h}$. Aliquots of $10 \mathrm{~mL}$ of culture were collected at $0,30,60,80,100,120,140,160,180,200,220$, and $240 \mathrm{~min}$. Cells were harvested by centrifugation at $4000 \times \mathrm{g}$ in Thermo Electron CL3R centrifuge for $10 \mathrm{~min}$ at $4{ }^{\circ} \mathrm{C}$. The cell pellets were resuspended in 0.4 $\mathrm{mL}$ of the lysis buffer (20 mM Tris- $\mathrm{HCl}, \mathrm{pH} 8.0,150 \mathrm{mM} \mathrm{NaCl}, 10 \%$ glycerol) and lysed by passing twice through a French press (1500 psi). The samples were clarified by centrifugation at $14000 \times \mathrm{g}$ for $30 \mathrm{~min}$ at $4{ }^{\circ} \mathrm{C}$ in an Eppendorf $5417 \mathrm{R}$ centrifuge. To determine the cleavage activity of TEVHis protease in cell lysates, $50 \mu \mathrm{L}$ of purified eGFPTL-REAChHis fusion protein (final concentration $50 \mathrm{nM}$ ) in $50 \mathrm{mM}$ Tris- $\mathrm{HCl}$ buffer, $\mathrm{pH} 7.5$, were added to the reaction mixture, containing $50 \mu \mathrm{L}$ of cell lysate, $15 \mu \mathrm{L}$ of $10 \times$ TEV protease buffer $(0.5 \mathrm{M}$ Tris$\mathrm{HCl}, \mathrm{pH}$ 8.0, 5 mM EDTA), $1.5 \mu \mathrm{L} 0.1 \mathrm{M}$ DTT, and $33.5 \mu \mathrm{L}$ of water. Enzymatic reactions were performed in 96-well microtiter plates at $30{ }^{\circ} \mathrm{C}$. Spectral changes in the emission of recombinant eGFP-TLREACh1 were mesaured by utilizing Molecular Devices Spectra Max M5 plate reader. The initial rates of enzymatic reaction were calculated using Graph Pad Prism version 4.0.

Analyte Sensitivity Assay. Cultures of E. coli TOP10 cells with plasmid pHWG640:eGFP-TL-REAChHis and a TEV protease expressing plasmid, either pSAL:RSTEVHis (riboswitch) or pSAL: RSTEVC151A (negative control), in LB medium, supplemented with $100 \mu \mathrm{g} / \mathrm{mL}$ ampicillin and $25 \mu \mathrm{g} / \mathrm{mL}$ chloramphenicol, were grown at $37{ }^{\circ} \mathrm{C}$ to an $\mathrm{OD}_{600}$ of $0.4-0.5$ and induced with $0.4 \%$ rhamnose for pHWG640:eGFP-TL-REAChHis expression. After 60 min of induction, cultures were treated with appropriate concentration of theophylline in DMSO. Cells were grown at $37^{\circ} \mathrm{C}$ with shaking at $225 \mathrm{rpm}$ for $6 \mathrm{~h}$. Cells were harvested and lysed as described above (Proteolytic Assay in Bacteria).
HPLC Analysis. HPLC determinations were performed with an Agilent-1100 HPLC system including a Hewlett-Packard Chemstation (HP/Agilent, Wilmington, DE). The HP Chemstation consisted of a quaternary gradient pump (G1312A binary pump) with a G1315A diode array detector, $6 \mathrm{~mm}$ flow cell, and G1315A fluorescence detector and an Agilent 1313A autosampler, a $25 \mu \mathrm{L}$ sample loop, and HP Chemstation software. Sample ultrafiltrate $(20 \mu \mathrm{L})$ was injected into the HPLC system. Separation by gradient elution of fluorescent proteins was performed on an Agilent 300SB-C8 reverse phase column $(250 \times$ $4 \mathrm{~mm})$ proceeded by an Agilent reverse phase guard column $(4 \times 12.5$ $\mathrm{mm}$ ). The mobile phase consisted of two components, A and B. Component A was prepared by mixing $1 \mathrm{~mL}$ of $100 \%$ TFA stock with $99 \mathrm{~mL}$ of deionized water. Component B $(0.09 \%$ TFA in $80 \%$ acetonitrile and $20 \%$ water) was prepared by mixing the ingredients in the given ratios. The mobile phase consisted of a linear gradient from an initial concentration of $100 \%$ A increasing up to $40 \%$ B in 25 min. The column effluent was monitored using an excitation wavelength of $457 \mathrm{~nm}$ and monitoring emission wavelengths of $510 \mathrm{~nm}$, corresponding to the fluorescence peak of eGFP. For linearity, the maximum output of the detector was limited to less than $10 \mathrm{LU}$ (light units) by appropriate dilution of the fluorescent proteins in the samples. The peak areas were integrated using the HP Chemstation integration software. Fluorescent protein concentrations were determined using their standard calibration curves. His-tagged fluorescent proteins eGFP-TL-REACh, REACh, and eGFP were expressed and purified in E. coli. Expression and purification of His-tagged proteins were carried out following standard protocols from QIAexpress Ni-NTA fast Start Handbook. The concentrations of the purified protein standards used for both REACh and eGFP were 1, 2, 5, 10, and $20 \mathrm{mg} / \mathrm{mL}$.

\section{Results and Discussion}

The fundamental strategy in this work utilizes a recognition element derived from in vitro aptamer screening to sense the small molecule theophylline, ${ }^{21}$ and in turn, this sensing event produces a cellular response, for example, a change in gene expression. ${ }^{23}$ The aptamer moiety of this riboswitch has been well-characterized and displays a 10000-fold higher affinity for theophylline when compared to structurally related caffeine. ${ }^{24}$ Desai and Gallivan were the first to demonstrate the theophylline-dependent activation of a reporter gene $\beta$-galactosidase in E. coli, utilizing an externally added substrate $\mathrm{X}$-gal to produce a color change in cells. ${ }^{23}$ In this work, we present an alternative reporting system based on an optical change, which will aid the ability to screen future synthetic riboswitches for novel analytes. Our studies involve the creation of a signal amplification assay via the enzymatic cleavage of an optically engineered substrate. E. coli cells produced a FRET-based protein which displayed a uniquely characteristic low optical background. We observed an increase in fluorescent signal in response to the presence of theophylline via riboswitch activation of TEV protease translation in an extremely low optical background, which allowed us to increase analyte threshold sensitivity.

Optical Reporter Assay to Screen for Synthetic Riboswitch Activity. Our novel optical assay for the readout of riboswitch activation was composed of TEV protease and its FRET-based engineered protein substrate, eGFP-TL-REACh. Representative emission spectra of purified eGFP-TL-REACh both cleaved and uncleaved by TEV protease is shown in Figure 2. The eGFP-TL-REACh protein displays extremely low fluorescence intensity due to the energy absorbing properties of the REACh chromophore. When eGFP-TL-REACh protein is cleaved by TEV protease, the fluorescence emission of eGFP observed at $510 \mathrm{~nm}$ is increased due to release of the eGFP chromophore from close proximity to the REACh protein. Therefore, riboswitch activation by theophylline was analyzed 


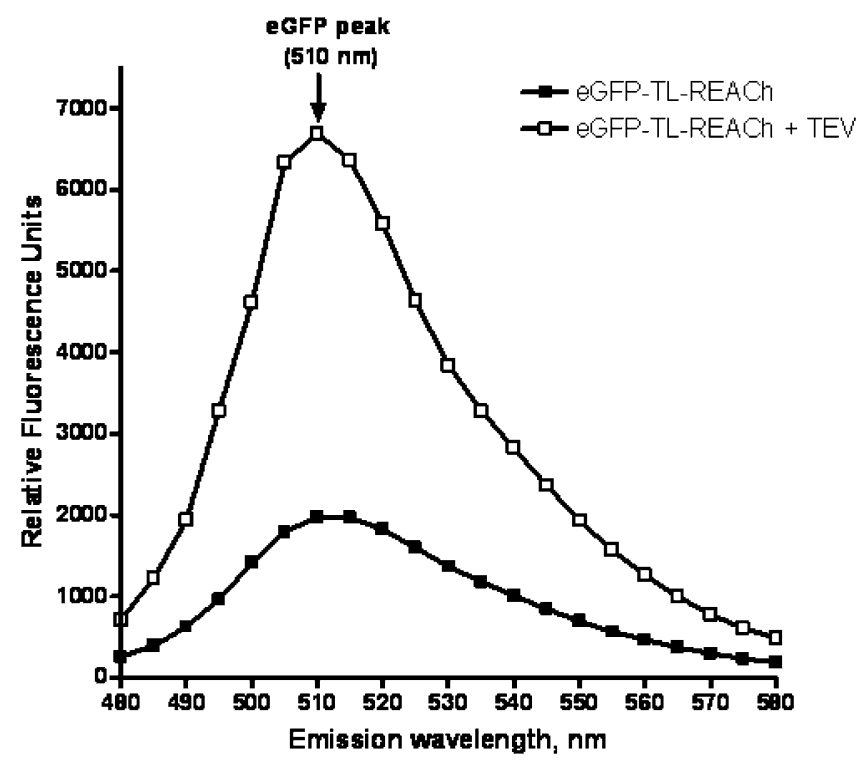

Figure 2. Cleavage of recombinant eGFP-TL-REACh FRET-based protein in vitro. The eGFP-TL-REACh construct was incubated with $0.5 \mu \mathrm{g}$ of recombinant TEV protease per $\mu \mathrm{g}$ of protein for $16 \mathrm{~h}$ (white squares) or without TEV protease (black squares). Emission spectra are shown for excitation at $457 \mathrm{~nm}$, which is optimal for eGFP. Arrow indicates the eGFP emission peak after the dark eGFP-TL-REACh protein is cleaved with TEV protease.

in relationship to the increase in eGFP fluorescence which is proportional to the cleavage of eGFP-TL-REACh by TEV protease.

To confirm the theophylline-dependent increase in TEV protease activity, cell cultures harboring synthetic riboswitch were compared to control cell cultures without riboswitch. The relative change in fluorescent signal, measured at the eGFP emission wavelength of $510 \mathrm{~nm}$, showed a riboswitch-dependent 2 -fold increase in TEV activity relative to the control cell culture (as shown in Figure SI.1). Changes in the spectral profiles of these cultures were attributed to riboswitch behavior and do not result from variation in their growth pattern. The cell growth curves were similar (overlap shown in Figure SI.2) for all culture conditions, which correlates well with the findings that theophylline below $5 \mathrm{mM}$ is nontoxic to the cells. Therefore, speculations about theophylline-induced changes in cell viability were eliminated.

The harvested cell extract showed greater signal amplification than the cell cultures due to the spectral interference of the medium. As shown in Figure 3, we measured riboswitch activation over time via the increase in fluorescence at $510 \mathrm{~nm}$. The TEV protease under riboswitch control displayed an 11.3fold increase in signal in the presence of theophylline over the riboswitch "off" state. The "off" state presented a similar fluorescence profile as the negative control culture, which produced a mutant, that is, inactive TEV protease; thus, the "off" state of the riboswitch mimics the behavior (spectral profile) of the negative control. The positive control, which harbored TEV protease gene without the riboswitch, showed the maximum signal output for this in vivo screening approach limited by TEV protease production in the cells, specifically a 2-fold higher signal than cells expressing riboswitch. Although we achieved maximum signal output in the presence of higher analyte concentrations, we did not expect to observe a $100 \%$ activation of the RS-TEV protease transcript, due to conformation restrictions in the 5'-UTR riboswitch structure. Gallivan and coworkers have determined that, in their riboswitch-containing

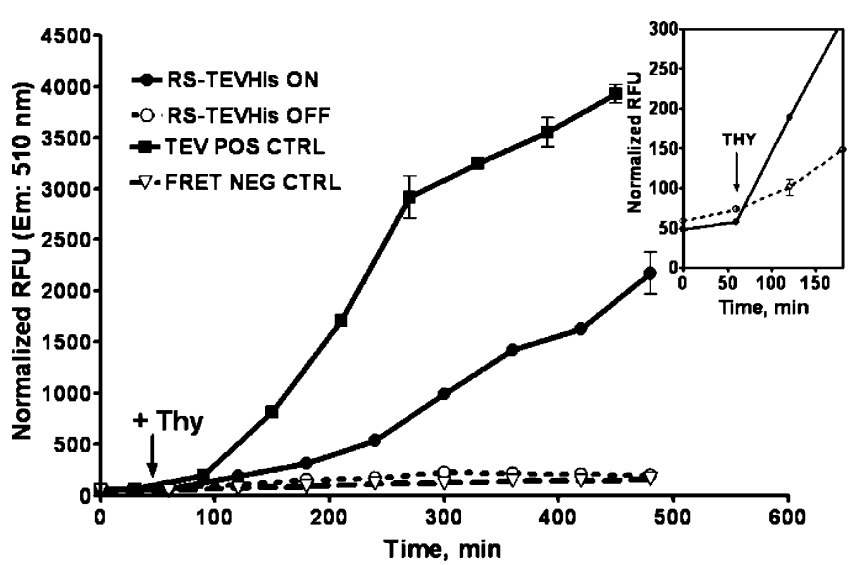

Figure 3. Time course profiles of riboswitch activation in response to theophylline addition. The riboswitch "off" and "on" states are displayed in terms of TEV protease activity in response to no small molecule (white circles) and $2.5 \mathrm{mM}$ theophylline at 60 min (black circles), respectively. The positive control shows TEV protease activity which is not modulated by the riboswitch at the 5'-UTR (black squares), i.e. constitutively on. The FRET eGFP-TL-REACh negative control (harboring inactive TEV protease) displays background levels of fluorescence, providing a low optical background without cleavage of the FRET protein (inverted triangles). (Inset) The trend lines for the "on" and "off" riboswitch states diverge after the addition of analyte.

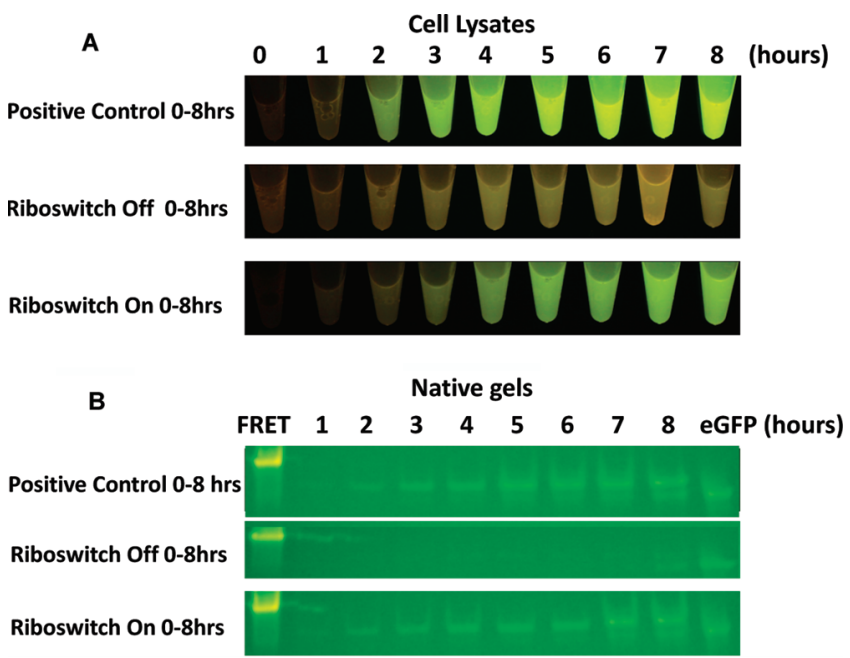

Figure 4. Visualization of TEV protease activity as seen by the increase in the fluorescence signal in cell lysates and the appearance of eGFP on nondenaturing polyacrylamide gel. Aliquots of cultures were harvested hourly pre- and post-theophylline exposure. (A) Clarified lysates display a fluorescent signal increase due to the TEV protease cleavage of the eGFP-TL-REACh protein. (B) $12 \%$ Tris$\mathrm{HCl}$ nondenaturing PAGE shows purifed FRET eGFP-TL-REACh $(5.7 \mu \mathrm{g})$ in the first lane, purified eGFP $(0.8 \mu \mathrm{g})$, and aliquot of each lysate (36 $\mu \mathrm{g}$ of total protein) for each time point over $8 \mathrm{~h}$ as indicated above. These nondenaturing PAGE have been stained with Coomassie blue (Figure SI.4).

construct, $16 \%$ of the transcript is in an active conformation, leaving $84 \%$ inactive. $^{21}$ The benefit for this conformational limitation is that it confers more translational control, yielding fewer false positives and lower background.

We observed marked divergence of signal as early as $60 \mathrm{~min}$ post-theophylline addition as shown by the inset graph in Figure 3. Due to low optical background, early activation of the riboswitch upon theophylline exposure was visually confirmed, as shown in Figure 4. The cleavage of the FRET protein in cellular extract was observed using an ultraviolet light source to reveal a well-defined band in nondenaturing polyacrylamide 


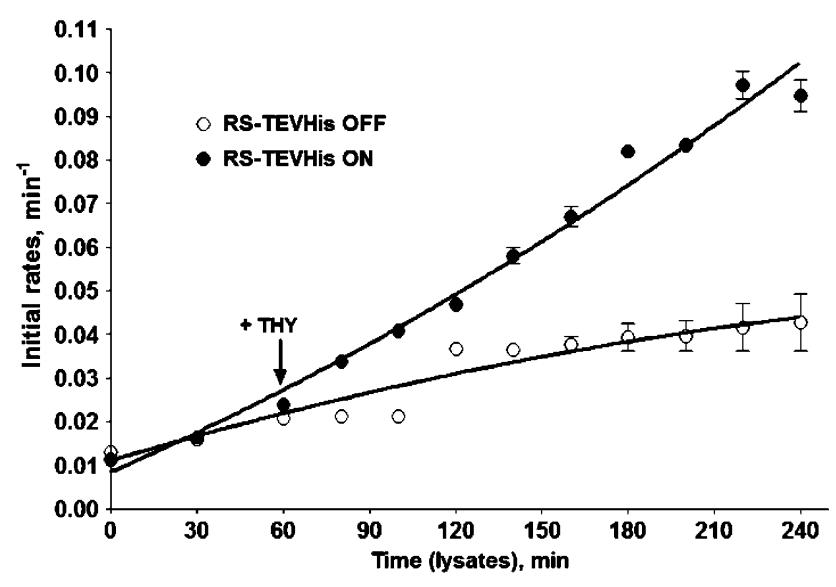

Figure 5. Kinetics of riboswitch-TEV protease activity from cellular extracts. TEV protease activity examined with $50 \mathrm{nM}$ eGFP-TLREACh. The riboswitch "off " and "on" states are displayed in terms of TEV protease activity in response to no analyte (white circles) and $2.5 \mathrm{mM}$ theophylline at $60 \mathrm{~min}$ (black circles), respectively. The positive control is shown in Figure SI.5.

gel electrophoresis. Cleavage of the EGFP-TL-REACh substrate corresponds to the increase in intensity of the eGFP band in both the activated riboswitch sample ( + theophylline) and the positive TEV protease control samples. We also observed an absence of the eGFP band in the riboswitch "off" cell extract even after $8 \mathrm{~h}$ of incubation with theophylline, thus indicating that TEV protease expression is tightly regulated by the riboswitch in this vector system.

Riboswitch Characterization. To determine the dose response time and eliminate both the burden and the variability on the cell to make the eGFP-TL-REACh protein, riboswitch activity was characterized directly by examining TEV protease expression via collected cell lysates. The TEV protease activity as a function of culture time was measured via an enzyme activity assay with a known concentration of FRET eGFP-TLREACh substrate. Coexpression of TEV protease and the FRET substrate did not affect TEV protease expression when compared to the TEV-only expressing cells. The TEV protease activity showed a similar response time, as did the cultures expressing both the FRET pair and the protease. In Figure 5, the riboswitchdependent TEV protease expression was initiated upon the addition of theophylline. The TEV activity curves for "on" and "off" states of the riboswitch begin to diverge after theophylline addition, indicating the increase in TEV protease in the presence of theophylline. The TEV protease increase over time was also confirmed using Western blot analysis (as shown in Figure SI.3). Thus, activation of the riboswitch was indicated by the increase in TEV protease activity determined over the time course of the collected cell cultures. The TEV protease concentration was 2 -fold greater in the riboswitch "on" state than that of the riboswitch "off" state. As indicated in both the Western blot and activity analyses, the TEV protease yield in response to riboswitch activation was measured in the range of 200-300 $\mathrm{nM}$, whereas the positive control without riboswitch produced 500-600 nM of TEV protease. This significantly lower yield of protein resulting from riboswitch activation demonstrates the benefit of further enzymatic amplification of riboswitch-driven signal as an indicator of analyte presence.

Quantification of the Optical Signal. To quantify more accurately the theophylline-dependent increase in TEV protease activity, the eGFP protein levels in E. coli clarified lysate samples were determined using an HPLC method coupled with fluorescence detection. When E. coli clarified lysates were used,

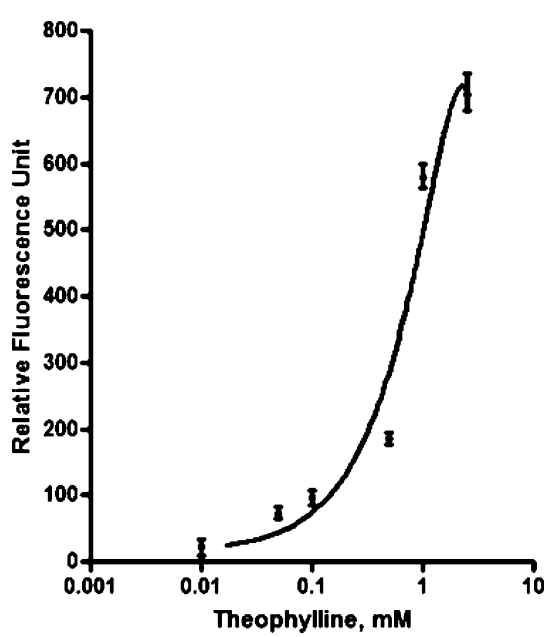

Figure 6. Theophylline response curve to determine the threshold of detection. The relative fluorescent units were divided by total protein concentration at $6 \mathrm{~h}$ post-theophylline exposure.

the lower detection limit for eGFP was calculated to be 1.76 $\mathrm{mg} / \mathrm{mL}$. This was determined using an eGFP standard curve extrapolated through 0 (as shown in Figure SI.6). The cultures harboring the synthetic riboswitch in the presence of theophylline showed an 11.3-fold increase in eGFP protein levels compared to the riboswitch "off" state and the negative control culture containing the inactive mutant TEV protease, when correlated (normalized) with total protein concentration of the cellular extracts. The eGFP protein levels in the "off" state and the negative control cultures were measured near the lower limit of detection. Also the HPLC data confirmed the TEV protease expression without the riboswitch, that is, positive control, yielded a 2-fold greater concentration of eGFP signal compared to expression under riboswitch translational control.

Improvement in Analyte Sensitivity. In order to investigate thresholds of detection, we have examined the fluorescence signal response in terms of small molecule concentration. As depicted in Figure 6, we measured the linear fluorescent response of the reporter as a function of analyte concentration, demonstrating a typical dose-response curve. Measuring optical response at higher analyte concentrations, that is, saturation, was impossible due to the toxic effects of theophylline at the higher concentrations. The lower concentrations of analyte are distinguishable due to the low optical starting point, that is, minimal optical background. The low optical background of the eGFP-TL-REACh construct allowed for a more dynamic range of analyte detection. The REACh chromophore eliminates the contribution of "the donor emission bleed-through and direct acceptor excitation".

The higher signal-to-noise attribute of this reporter system is depicted in Figure 7 where one can see the clear difference in the cellular response to the analyte even in the presence of media interference. The dramatic signal improvement can be seen in the graph, which shows the large dynamic range in signal intensity observed in the cellular extracts. The optical read-out of this system is theoretically limitless in terms of spectral output, however, cellular systems have their own protein production constraints.

The amplification of signal via TEV protease cleavage is a benefit, however the additional requirement of cells producing the protease delays signal response to the analyte. In some settings, a time delay has applications. Reduction of this time delay may be achieved with direct expression of a fluorescent protein as shown by Smolke and co-workers. ${ }^{12}$ However, the 


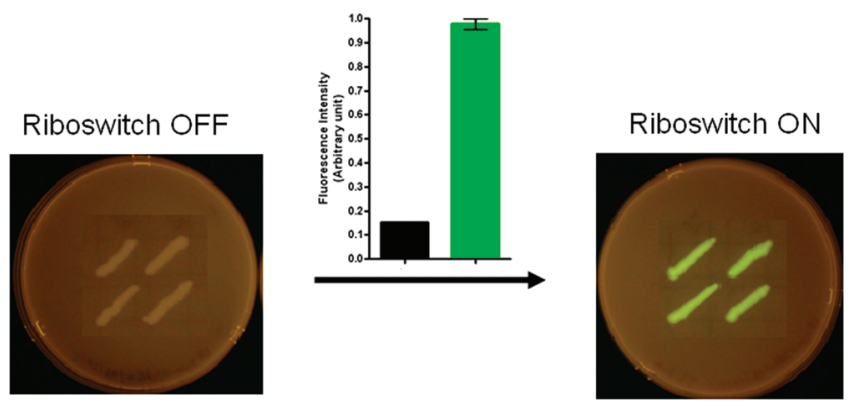

Figure 7. Demonstration of optical assay improvement in cellular extracts. The REACh-based FRET construct produced an 11.3-fold difference in the optical signal between "on" (green bar) and "off" (black bar) riboswitch states.

fluorescence from the produced protein must be of sufficient quantity and intensity to produce a quantitative response signal to analyte. In our assay, when the eGFP gene is placed directly downstream of the riboswitch in the pSAL vector, we observed an extremely low expression of eGFP and activation of the riboswitch as shown in terms of fluorescence increase (data shown in Figure SI.7). Thus, in our assay, lower analyte concentration is more readily detected using the eGFP-TLREACh construct because of the lower initial optical background and the enzymatic activity of the TEV protease.

\section{Conclusions}

We have developed a novel optical reporter assay for monitoring riboswitch activation, which is based on fluorescence resonance energy transfer between the eGFP and REACh protein pair. The REACh protein in this construct acts as a quencher, eliminating the acceptor fluorescence and providing greater quantitative analysis of the output signal. We have demonstrated the performance of a new reporter system in E. coli cells by monitoring the activation of synthetic theophylline riboswitch placed upstream of the TEV protease gene. In the absence of theophylline, when the riboswitch is in the "off" state and prevents the translation of TEV protease, the REACh protein quenches the fluorescent signal thus producing very low background fluorescence. Addition of theophylline changes the conformation of the riboswitch and initiates the translation of mRNA. Synthesized TEV protease cleaves the FRET-proteins yielding an 11.3-fold increase in fluorescence level in the presence of theophylline over the riboswitch "off" state in cellular extract. Quantitative HPLC analysis of eGFP levels in $E$. coli extract confirmed this signal fold change. The low background fluorescence and enzymatic amplification of the developed reporter assay allowed us to monitor riboswitch activation at analyte concentrations ranging from 0.01 to 2.5 $\mathrm{mM}$.

We have demonstrated the ability to monitor riboswitch activity utilizing an enzymatically triggered optical reporter protein. From a signal processing perspective, it is advantageous to use a low optical background assay. In this work, we have exerted temporal control and specificity by incorporating a riboswitch upstream of the protease. This riboswitch-driven protease construct cleaves a protein substrate, yielding a high optical output over several orders of magnitude in analyte concentration.

Acknowledgment. We acknowledge the efforts of Sandra Dias and John Lynch. This work was supported by funds provided by the Air Force Office of Scientific Research (AFOSR) and by the U.S. Department of Defense High Performance Computing Modernization Program (HPCMP), under the High Performance Computing Software Applications Institutes (HSAI) initiative.

Supporting Information Available. Experimental details and analytical results. This material is available free of charge via the Internet at http://pubs.acs.org.

\section{References and Notes}

(1) Mandal, M.; Breaker, R. R. Nature Rev. Mol. Cell. Biol. 2004, 5, 451463.

(2) Tucker, B. J.; Breaker, R. R. Curr. Opin. Struct. Biol. 2005, 15, 342348.

(3) Nudler, E.; Mironov, A. S. Trends Biochem. Sci. 2004, 29, 11-17.

(4) Winkler, W. C. Curr. Opin. Chem. Biol. 2005, 9, 594-602.

(5) Winkler, W.; Nahvi, A.; Breaker, R. R. Nature (London) 2002, 419, 952-956.

(6) Werstuck, G.; Green, M. R. Science 1998, 282, 296-298.

(7) Grate, D.; Wilson, C. Bioorg. Med. Chem. 2001, 9, 2565-2570.

(8) Harvey, I.; Garneau, P.; Pelletier, J. RNA 2002, 8, 452-463.

(9) Bauer, G.; Suess, B. J. Biotechnol. 2006, 124, 4-11.

(10) Silverman, S. K. Artificial functional nucleic acids: aptamers, ribozymes and deoxyribozymes by in vitro selection. In Functional Nucleic Acids for Analytical Applications; Li, Y. , Lu, Y., Eds.; Springer: Berlin, 2009.

(11) Isaacs, F. J.; Dwyer, D. J.; Collins, J. J. Nat. Biotechnol. 2006, 24, $545-554$.

(12) Win, M. N.; Smolke, C. D. Proc. Natl. Acad. Sci. U.S.A. 2007, 104, 14283-14288.

(13) Hanson, S.; Bauer, G.; Fink, B.; Suess, B. RNA 2005, 11, 503-511.

(14) Weigand, J. E.; Sanchez, M.; Gunnesch, E.-B.; Zeiher, S.; Schroeder, R.; Suess, B. RNA 2008, 14, 89-97.

(15) Kohl, T.; Heinze, K. G.; Kuhlemann, R.; Koltermann, A.; Schwille, P. Proc. Natl. Acad. Sci. U.S.A. 2002, 99, 12161-12166.

(16) Förster, T. Ann. Phys. 1948, 2, 55-75.

(17) Ganesan, S.; Ameer-beg, S. M.; Ng, T. T. C.; Vojnovic, B.; Wouters, F. S. Proc. Natl. Acad. Sci. U.S.A. 2006, 103, 4089-4094.

(18) Kapust, R. B.; Tozser, J.; Fox, J. D.; Anderson, D. E.; Cherry, S.; Copeland, T. D.; Waugh, D. S. Protein Eng. 2001, 14, 993-1000.

(19) Lucast, L. J.; Batey, R. T.; Doudna, J. A. BioTechniques 2001, 30, $544-554$.

(20) van den Berg, S.; Löfdahl, P.-A.; Härd, T.; Berglund, H. J. Biotechnol. 2006, 121, 291-298.

(21) Lynch, S. A.; Desai, S. K.; Sajja, H. K.; Gallivan, J. P. Chem. Biol. 2007, 14, 173-184.

(22) Sambrook, J.; Russell, D. W. Molecular Cloning: A Laboratory Manual, 3rd ed.; Cold Spring Harbor Laboratory Press: Cold Spring Harbor, NY, 2001.

(23) Desai, S. K.; Sajja, H. K.; Gallivan, J. P. J. Am. Chem. Soc. 2004, 126, 13247-13254.

(24) Jenison, R. D.; Gill, S. C.; Pardi, A.; Polisky, B. Science 1994, 263, $1425-142$.

BM801117F 